\title{
Proyección Cónica Equivalente de Albers: propiedades y aplicación para México
}

\author{
Mariana Vallejo Velázquez* \\ Jesús Abraham Navarro Moreno**
}

Recibido el 01 de marzo de 2016: aceptado el 17 de noviembre de 2016

\begin{abstract}
The national mapping of Mexico employs three map projections: the Universal Transverse Mercator, for large-scale and medium-scale topographic maps; the Lambert Conformal Conic, for mapping the country in small-scale maps; and the Mercator projection used for nautical charts. The usual employment of these projections has been recognized by the scientific community so they are normally used; however, in Mexico the cartographic research related to this subject has not been produced, and has not sought the application of other map projections in an effort to achieve a better representation of the results of the various spatial research topics. This paper aims to show the use of the Albers Equal-Area Conic projection and its parameters. In this sense, it shows the formulations that allows the projection of the coordinates, the analysis of the distortions and the conception of national mapping in a 1:500,000 scale maps; thus, the article intends to encourage the reflection about the non-conformal characteristic for Mexican mapping.

Key words: Mathematical Cartography, equal-area projection, Albers EqualArea Conic, Mexico.
\end{abstract}

\section{Resumo}

A cartografia nacional do México é feita a través do uso de três projeções oficias: Universal Transversa de Mercator, utilizada em mapas topográficos de escalas grande e media; Cônica Conforme de Lambert, adaptada às condições do país para sua representação em escalas pequenas; e a Normal de Mercator, utilizada para fins de navegação marítima. O uso destas projeções a longo do tempo foi assimilado

* Estudiante de especialidad en Geomática en el Centro de Investigación en Geografía y Geomática "Ing. Jorge L. Tamayo", A.C., correo electrónico:mvv_geo@hotmail.com

** Colegio de Geografía, Facultad de Filosofía y Letras, Universidad Nacional Autónoma de México, correo electrónico: a.navarro@comunidad.unam.mx. 
pela comunidade científica e usado de forma habitual; no entanto, a pesquisa cartográfica relacionada com esta temática parou e não se tem procurado a aplicação de outras projeções como um esforço para alcançar uma melhor representação dos vários tópicos de pesquisa, o que também afeta a precisão dos dados obtidos nestas investigações. Este trabalho tem como objetivo mostrar a utilização da projeção Cônica Equivalente de Albers com as adaptações dos parâmetros exigidos; a este respeito, presentam-se as formulações que permitem a projeção das coordenadas, a análise das deformações e o mapeamento de uma cartografia nacional escala 1:500.000; posteriormente, o trabalho também tem o objetivo de estimular a reflexão sobre as possibilidades da projeção que não preserva o ortomorfismo, considerado até agora como a única propriedade na cartografia nacional mexicana.

Palavras chave: Cartografia Matemática, projeção cartográfica equivalente, Cônica Equivalente de Albers, México.

\section{Resumen}

La cartografía nacional de México emplea de manera oficial tres proyecciones: la Universal Transversa de Mercator, utilizada en las cartas topográficas para escalas medianas y grandes; la Cónica Conforme de Lambert, adaptada a las condiciones del país para su representación en escalas pequeñas; y la Normal de Mercator, empleada con fines de navegación marítima. El uso por largo tiempo de estas proyecciones ha sido asimilado por la comunidad científica y se emplean de forma regular; no obstante, la investigación cartográfica relacionada con esta temática se ha detenido y no se ha buscado la aplicación de otras proyecciones cartográficas en el afán de alcanzar una mejor representación de los diversos temas de investigación, lo que impacta también en la precisión de los datos obtenidos. El presente trabajo tiene por objetivo mostrar el empleo de la proyección Cónica Equivalente de Albers con las adaptaciones de parámetros necesarios; en este sentido, se presentan las formulaciones que posibilitan la proyección de las coordenadas, el análisis de las deformaciones y la concepción una cartografía nacional a escala 1:500,000; subsecuentemente, el trabajo también pretende estimular la reflexión en torno a las posibilidades que tiene esta proyección que no preserva el ortomorfismo, considerado hasta ahora como única propiedad en la cartografía nacional mexicana.

Palabras clave: Cartografia Matemática, proyección cartográfica equivalente, Cónica Equivalente de Albers, México.

\section{Introducción}

Un mapa requiere de un alto grado de afinidad con la realidad; debe ser confiable, legible y geométricamente preciso (Eckert, 1961; Salitchev, 1979; Franco y Valdez, 2003), ya que la representación equivocada de la información conducirá a una in- 
terpretación errada de la realidad. La precisión geométrica, particularmente, deriva de la base matemática del mapa, que está conformada por la escala, el sistema de coordenadas y la proyección cartográfica (Franco y Valdez, op. cit.).

En lo concerniente a las proyecciones cartográficas, puede pensarse que el problema de transferir la forma curva de la Tierra a un plano de representación está esencialmente resuelto por la ciencia matemática, por consecuencia, no es necesario formular más proyecciones; por otro lado, en tanto se mantengan las convenciones y parámetros establecidos, la decisión con respecto a la elección de la proyección para diseñar un mapa es una tarea sencilla.

Asumir esta postura, sin embargo, disminuye en gran medida las reflexiones en torno a la Cartografía Matemática y los aportes que esta vertiente investigativa tiene para plasmar la superficie terrestre. Los realizadores de mapas que se sitúan bajo esta visión, comúnmente eligen entre un número reducido de proyecciones, lo que deriva en la homogeneización de las representaciones; pero más allá, se puede pensar que sólo es un requerimiento para obtener información georreferenciada.

La elección de una proyección cartográfica, ya sea para hacer un mapa o planificar una cartografía nacional, se vincula directamente con las bases teóricas de la Cartografía, y es una tarea de suma importancia porque induce la manera en que es visualizada la información; al respecto, Dent (1999) señaló que cada mapa temático tiene una proyección, y entender esto debería ser parte de la educación de un diseñador de mapas.

Asimismo, si se reflexiona en la perspectiva práctica del uso de los mapas, la proyección cartográfica también influye en los resultados que se pueden obtener al realizar cálculos analíticos o mediciones cartométricas.

\section{Perspectivas investigativas relacionadas con el estudio de las proyecciones cartográficas}

En los últimos años, las investigaciones sobre proyecciones cartográficas se han centrado principalmente en tres enfoques: deformaciones, formulaciones de nuevas proyecciones y propuestas de proyecciones cartográficas para mapas en Internet. Con respecto al estudio de las deformaciones, los trabajos se encaminan a desarrollar métodos de cálculo de los valores de deformación de las proyecciones y la forma gráfica de su representación (Kirtiloglu, 2010; Battersby y Kessler, 2012; Oztug, 2015). Por su parte, las perspectivas dedicadas a la propuesta de nuevas proyecciones buscan reducir en la medida de lo posible las deformaciones; la tendencia es desarrollar proyecciones de manera visual (visually designed projections) con el uso de softwares, esta práctica permite que usuarios no profesionales en la materia puedan crear sus propias proyecciones; las proyecciones que se formulan se fundamentan en las desarrolladas matemáticamente y, generalmente, son resultado de la combinación de dos proyecciones (Alashaikh, et al., 2014; Jenny y Patterson, 
2013), aunque siguen surgiendo nuevas con parámetros distintos (Šavrič, et al., 2011; Jenny, et al., 2011; Safari y Ardalan, 2007; Paterson, et al., 2015). Finalmente, otros estudios se están encaminando en proponer nuevas proyecciones para mapas desplegados en los visualizadores dinámicos de Internet; el argumento se centra en que la proyección de Mercator, empleada en la web, presenta desventajas en el manejo de la información a diferentes escalas (Šavrič y Jenny, 2014; Streben, 2016).

El número de trabajos de investigación referente al análisis de proyecciones cartográficas representan los esfuerzos que existen alrededor del mundo, en especial en Estados Unidos, por desarrollar representaciones más precisas de la superficie terrestre.

En México no se ha encontrado bibliografía que evidencie continuidad de las investigaciones sobre Cartografía Matemática; los estándares y normas que rigen la elaboración de cartografía nacional oficial se basan en postulaciones de larga historia en el país. Paquentín (1956) determinó que la proyección Cónica Conforme de Lambert era adecuada para la representación de México; por su parte Caire (1974) recomendó el empleo de la proyección Universal Transversa de Mercator (UTM) para la elaboración de cartografía nacional y presentó sus formulaciones; en años más recientes, Raúl A. Gómez elaboró una guía de proyecciones cartográficas (Instituto Nacional de Estadística y Geografía - INEGI, 2002), que además de incluir las proyecciones que oficialmente se utilizan para México, desarrolló cálculos y ejercicios para la transformación de coordenadas en siete proyecciones más, aunque sin particularizar adaptaciones específicas para el territorio de México.

De forma posterior a la publicación de estas investigaciones, no ha habido propuestas conocidas que esgriman el empleo de otras proyecciones y que pudieran ser favorables para la representación del territorio mexicano.

\section{Proyecciones cartográficas empleadas para México}

El INEGI es la institución oficial que establece las especificaciones para la cartografía nacional. La norma NTG-013-2006 Edición de Cartografía Topográfica (INEGI, 2006) regula la edición cartográfica para normalizar formatos, escalas y simbologías, en el apartado seis menciona las descripciones técnicas y parámetros de las tres proyecciones cartográficas empleadas para la elaboración de cartas topográficas (véase Tabla 1).

La CCL es una proyección conforme, secante y normal, se caracteriza por mostrar los paralelos como arcos de circunferencias no equidistantes concéntricas al vértice del cono, la distancia entre ellos disminuye al centro de la proyección. Los meridianos son radios rectos equidistantes que con los paralelos forman perpendiculares. Como fue mencionado anteriormente, en 1956 Eduardo Paquentín, de la entonces Secretaría de Recursos Hidráulicos (actualmente Comisión Nacional del 
Agua), concluyó que entre la proyección Transversa de Mercator, la Cónica Conforme de Lambert y la Estereográfica, la CCL era la más adecuada para representar al territorio mexicano en escalas pequeñas (Alonso, 1989). En este sentido, se puede apreciar que la preferencia por el uso de la CCL se basa en la comparación con respecto a una perspectiva acimutal y otra cilíndrica.

Tabla 1

Proyecciones cartográficas empleadas para México por escala

\begin{tabular}{ll}
\hline \multicolumn{1}{c}{ Proyección cartográfica } & \multicolumn{1}{c}{ Escala } \\
\hline Cónica Conforme de Lambert (CCL) & $1: 4,000,000$ \\
Transversa de Mercator & $1: 1,000,000$ \\
Cónica Conforme de Lambert & $1: 500,000$ o mayores \\
Universal Transversa de Mercator (UTM)
\end{tabular}

Fuente: INEGI, 2006.

La proyección UTM es una proyección modificada por el U.S. Army Map Service en 1940 de la proyección Transversa de Mercator (Ibíd.), es una proyección y un sistema de coordenadas cartesianas métricas. La diferencia entre ambas es que la Transversa de Mercator es tangente y la UTM es secante (Robinson, et. al., 1987). En 1951 la Comisión Cartográfica Militar adoptó la UTM, que sustituyó a la proyección policónica, para construir la Carta General de la República Mexicana escala 1:100,000. En 1968 se creó la Comisión de Estudios del Territorio Nacional (CETENAL), hoy INEGI, que elaboró cartografía nacional base empleando esta proyección (Caire, op. cit.).

De manera adicional a las proyecciones consideradas por el INEGI, también se utiliza de forma oficial para México la proyección Normal de Mercator. Esta proyección se desarrolló con fines náuticos y muestra los rumbos como líneas rectas que indican la dirección como una constante lo que facilita la navegación. En México, la Secretaría de Marina-Armada de México (SEMAR) es la institución encargada de elaborar las cartas náuticas del país.

$\mathrm{Si}$ bien todas estas son proyecciones que se adecuan al territorio, es importante hacer énfasis en que todas son conformes, por lo que la cartografía oficial de México se caracteriza por esta propiedad; en este sentido, se puede proponer la aplicación de otro tipo de proyección. Los estudios espaciales, ya sean geográficos, topográficos, biológicos, ambientales, económicos, demográficos o de cualquier otra ciencia cuyo objeto de estudio sea la superficie terrestre, poseen un extenso campo de investigación, lo que se manifiesta en una amplia pluralidad de temas que se pueden abordar dentro de cada dimensión espacial. Todo tipo de estudio espacial posee características propias cuyos resultados pueden ser representados en un mapa 
$\mathrm{y}$, en consecuencia, cada mapa puede hacer uso de una base matemática cartográfica particular; por esto, se requiere reflexionar en las características de las proyecciones cartográficas, no sólo conformes, también equivalentes, equidistantes y anafilácticas.

\section{Proyecciones cartográficas equivalentes}

Las propiedades de las proyecciones equivalentes son de gran utilidad para temas espaciales. También llamadas equiárea o autálicas, tienen la propiedad de mantener en proporción el tamaño de una superficie; para lograr esta equivalencia, ocurre un proceso de compresión y extensión, y los ángulos de las figuras que se sitúen sobre la superficie se deforman (Tyner, 2010). Otra característica de este tipo de proyecciones es que el espaciado entre los paralelos y meridianos debe ser proporcional a la exageración que se tenga de ellos en el mapa.

Las proyecciones equivalentes son muy importantes para los mapas temáticos cuantitativos, porque el área, frecuentemente, es parte de los datos a ser incorporados en la representación; en este sentido, tienen una utilidad general, sin importar tanto las dimensiones que tenga el territorio cartografiado (Dent, op. cit.). Derivado de lo anterior, tienen más facilidad de aplicarse en un rango mayor de escalas.

Algunos casos que implican el uso de este tipo de proyección son:

- Mapas de densidad o concentración territorial que muestran la relación entre el número de objetos por unidad de superficie. El objetivo de este tipo de mapas es exponer al usuario, en una primera impresión, la cantidad de elementos que habitan en un espacio.

- Mapas en los que se emplean los símbolos proporcionales como método de representación. Mostrar el área a escala admite una lectura más certera de la información al permitir al usuario observar la relación entre el tamaño de la superfície y el tamaño del símbolo.

- Estudios que impliquen el reconocimiento de zonas de influencia.

- Estudios de cambios espacio-temporales requieren del empleo de una proyección equivalente que permita comparar las áreas afectadas y la rapidez con la que han ocurrido las trasformaciones espaciales.

- Estudios que requieran cálculos de áreas como son de usos de suelo, predios, cuencas hidrográficas, zonas de riesgo, municipios o regiones climáticas, entre otros.

No hay duda de la importancia de la cartografía equivalente; especialmente para los mapas de corte investigativo, la preservación de un área verdadera es más importante que el sostenimiento de un valor angular teórico. Marschner, 1944 (citado en Dent, op. cit.) argumentó a favor de la perspectiva equivalente en la Cartografía: 
el ser humano no habita en una línea de la Tierra, sino en áreas; tampoco se cultiva en un ángulo de la superficie, sino en un área.

En virtud de lo anterior, se puede proponer el uso de una proyección equiárea que favorezca las características del territorio mexicano. En general, para elegir una proyección se debe considerar: la extensión y geometría del territorio, el objetivo del mapa, los patrones de deformación y la escala del mapa; Robinson et al. (op. cit.) señalan que al elegir una proyección equivalente se debe considerar el tamaño del área a representar y la distribución de la deformación de los ángulos, ya que de ello depende la distorsión de la forma de la superficie.

\section{Proyección Cónica Equivalente de Albers}

La proyección Cónica Equivalente de Albers (CEA) (Figura 1) fue desarrollada en su forma esférica por el alemán Heinrich Christian Albers en 1805, posteriormente Oscar S. Adams presentó el desarrollo matemático para su forma elipsoidal (Snyder, 1997).

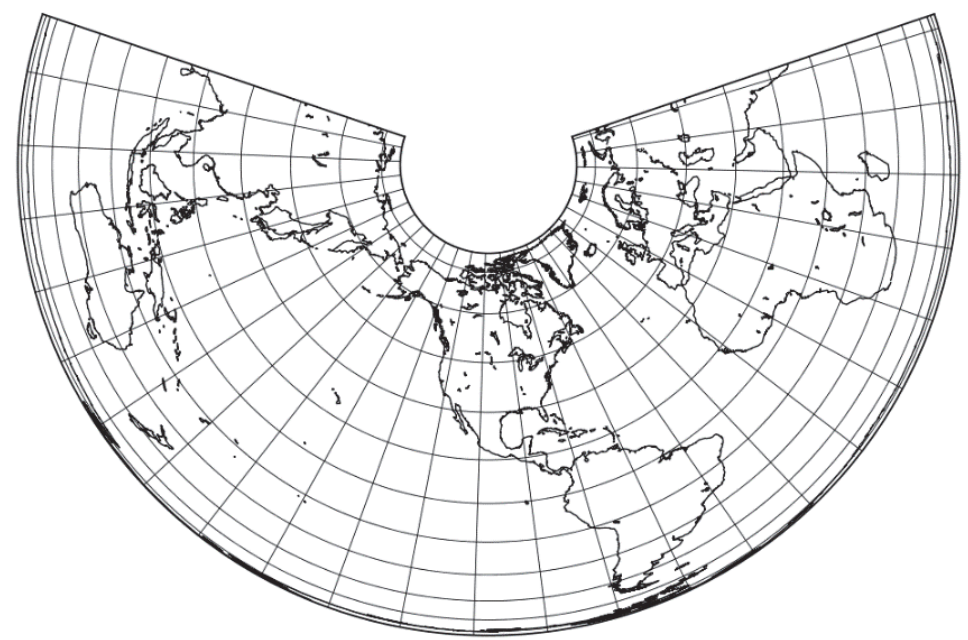

Figura 1. Proyección Cónica Equivalente de Albers.

Fuente: elaborado a partir de procesamiento en software.

Se trata de una proyección cónica secante — dos paralelos base- - y normal. Los paralelos y meridianos son ortogonales entre sí, los paralelos son arcos de circunferencias concéntricas no equidistantes, la distancia entre ellos disminuye a medida que se alejan de los paralelos base, por otro lado, los meridianos son líneas rectas equidistantes cuyo origen es el centro de las circunferencias. 
En una proyección se puede ubicar un punto con coordenadas sexagesimales o en valores métricos, las coordenadas métricas son las que van a permitir obtener cálculos geométricos de la superficie terrestre como áreas y distancias.

A continuación, se presentan las fórmulas empleadas y los procedimientos que explican la transformación directa - de coordenadas geodésicas a cartesianas- en proyección CEA (INEGI, 2002):

a. Calcular las latitudes de los paralelos base y el meridiano central

$$
\begin{gathered}
\varphi^{\prime} \text { sur }=\varphi \text { inferior }+\frac{\varphi^{\circ} \text { superior }-\varphi^{\circ} \text { inferior }}{j} \\
\varphi^{\prime} \text { norte }=\varphi \text { superior }-\frac{\varphi^{\circ} \text { superior }-\varphi^{\circ} \text { inferior }}{j} \\
\lambda_{o}=\frac{\lambda^{\circ} \text { este }-\lambda^{\circ} \text { oeste }}{2}
\end{gathered}
$$

Donde:

$\varphi=$ latitudes (grados y minutos) extremas de la zona a representar

$\varphi^{\circ} \lambda^{\circ}=$ grados de coordenadas extremas de la zona a representar

$j=$ constante. Para Kavrayskiy el valor de $j$ es 5 si la superficie a representar es similar a un rectángulo, 4 si es circular y 3 si es un rombo. Por otro lado, Deetz y Adams sugieren un valor constante de 6. Para México se aplica el valor de 6 para $j$

$\lambda_{0}=$ meridiano central

b. Con las latitudes de los paralelos base, obtener las constantes $m_{\text {norte }}, m_{\text {sur }} \mathrm{y}$ $m_{o}$ (latitud origen) de la proyección:

$$
e^{2}=\frac{a^{2}-b^{2}}{a^{2}}
$$




$$
\mathrm{m}_{\mathrm{i}}=\frac{\cos \varphi_{i}}{\sqrt{1-\mathrm{e}^{2} \operatorname{sen}^{2} \varphi_{\mathrm{i}}}}
$$

Donde:

$e^{2}=$ excentricidad al cuadrado.

$a=$ semieje mayor del elipsoide de referencia.

$b=$ semieje menor del elipsoide de referencia.

$\varphi_{i}=$ latitud de los paralelos base norte, sur y origen.

c. Obtener las constantes $q_{\text {norte }}, q_{\text {sur y }} q_{o}$ (latitud origen) de la proyección:

$$
\begin{gathered}
\boldsymbol{e}=\frac{\sqrt{a^{2}-b^{2}}}{a} \\
\left.\boldsymbol{q}_{\boldsymbol{i}}=\left(1-e^{2}\right)\left[\frac{\operatorname{sen} \varphi_{i}}{\left(1-e^{2} \operatorname{sen}^{2} \varphi_{i}\right.}\right)^{-\frac{1}{2 e}} \ln \left(\frac{1-e \operatorname{sen} \varphi_{i}}{1+e \operatorname{sen} \varphi_{i}}\right)\right]
\end{gathered}
$$

Donde:

$e=$ primera excentricidad.

d. Sustituir los valores obtenidos en los incisos B y C para resolver la constante del cono:

$$
\boldsymbol{n}=\frac{m_{\text {sur }}^{2}-m_{\text {norte }}^{2}}{q_{\text {norte }}-q_{\text {sur }}}
$$

e. Determinar $p_{i}$ para el paralelo origen $\left(p_{o}\right)$ :

$$
\boldsymbol{p}_{\boldsymbol{i}}=\frac{a \sqrt{m_{\text {sur }}^{2}+n\left(q_{\text {sur }}-q_{i}\right)}}{n}
$$

f. Para el cálculo de las coordenadas, calcular $q_{p}$ y $p_{p} \quad$ resolver las ecuaciones 7 y 9 , considerar la latitud del punto dado (en grados decimales). 
g. Obtener el valor de $\gamma$ y transformarlo a radianes:

$$
\begin{gathered}
\gamma=n\left(\lambda_{o}-\lambda_{p}\right) \\
\text { radianes }=\frac{\pi * \text { grados }}{180}
\end{gathered}
$$

Donde:

$\lambda_{p}=$ longitud del punto dado

h. Para obtener las coordenadas finales, calcular:

$$
\begin{gathered}
\boldsymbol{X}=E_{o}+p_{p} \operatorname{sen} \gamma \\
\boldsymbol{Y}=N_{o}+p_{o}-p_{p} \cos \gamma
\end{gathered}
$$

Donde:

$$
\begin{aligned}
& E_{o}=\text { falso Este } \\
& N_{o}=\text { falso Norte }
\end{aligned}
$$

El cálculo inverso - de coordenadas cartesianas a coordenadas geodésicas - en proyección CEA es semejante, y puede servir también a manera de comprobación de los cálculos directos. Para este proceso se resuelven las siguientes ecuaciones:

- Obtener el valor de las constantes $m_{\text {norte }}, m_{\text {sur }},\left(\right.$ ecuación 5), y $q_{\text {norte }}, q_{\text {sur }}$ (7) considerando las latitudes de los paralelos base.

- Calcular la constante del cono $n(8)$

- Obtener $p_{i}$ para el paralelo origen $p_{o}$ (9)

- Determinar los valores de $p, \gamma$ y $q$ :

$$
\begin{gathered}
\boldsymbol{p}=\sqrt{\left(E-E_{o}\right)^{2}+\left(p_{o}+N_{o}-N\right)^{2}} \\
\gamma=\arctan \left(\frac{E-E_{o}}{p_{o}+N_{o}-N}\right) \\
\boldsymbol{q}=\frac{\left(m_{\text {sur }}^{2}+n q_{\text {sur }}-\frac{p^{2} n^{2}}{a^{2}}\right)}{n}
\end{gathered}
$$


Donde:

$E$ y ${ }_{N}=$ valores de coordenadas $\mathrm{X}$ y $\mathrm{Y}$ del punto dado

$E_{o}$ y $N_{o}=$ valores de falso Este y falso Norte

$\gamma=$ una vez obtenido el resultado transformar a radianes (11)

- A continuación se desarrolla la primera aproximación de la latitud $\left(\varphi_{o}\right)$, este valor debe transformarse a radianes (11):

$$
\varphi_{\boldsymbol{o}}=\operatorname{arcsen}\left(\frac{q}{2}\right)
$$

A partir de esta se calcularán valores más precisos de la latitud con un proceso iterativo:

$$
\Delta_{\varphi}=\varphi_{i-1}+\frac{\left(1-e^{2} \operatorname{sen}^{2} \varphi_{i-1}\right)^{2}}{2 \cos \varphi_{i-1}}\left[\left(\frac{q}{1-e^{2}}\right)-\left(\frac{\operatorname{sen} \varphi_{i-1}}{1-e^{2} \operatorname{sen}^{2} \varphi_{i-1}}\right)+\frac{1}{2 e} \ln \left(\frac{1-\operatorname{esen} \varphi_{i-1}}{1+e \operatorname{sen} \varphi_{i-1}}\right)\right]
$$

La función iterativa concluye cuando se cumple la condición $\left|\varphi_{1}-\varphi_{i-1}\right| \leq 1 * 10^{-5}$ segundos, una vez cumplida se obtiene la latitud final.

- Para concluir se determina la longitud:

$$
\lambda=\lambda_{o}-\frac{\gamma}{n}
$$

El resultado de la división $\frac{\gamma}{n}$ está dado en radianes, por lo que se debe transformar a grados para resolver la sustracción.

Las características de la proyección CEA pueden favorecer la representación de la República Mexicana por dos motivos: en primera instancia, las proyecciones cónicas son aptas para la representación de territorios que se prolongan longitudinalmente (de Este a Oeste) debido a que el desarrollo del cono se extiende en este sentido; por otro lado, al ser una proyección secante, los dos paralelos base permiten una menor deformación latitudinal al centro de la proyección y en las latitudes extremas de México. Además de estos dos argumentos, esta proyección tiene la propiedad de conservar el área en correcta proporción sin deformar en gran medida la forma de los territorios (Figura 2). 
a)

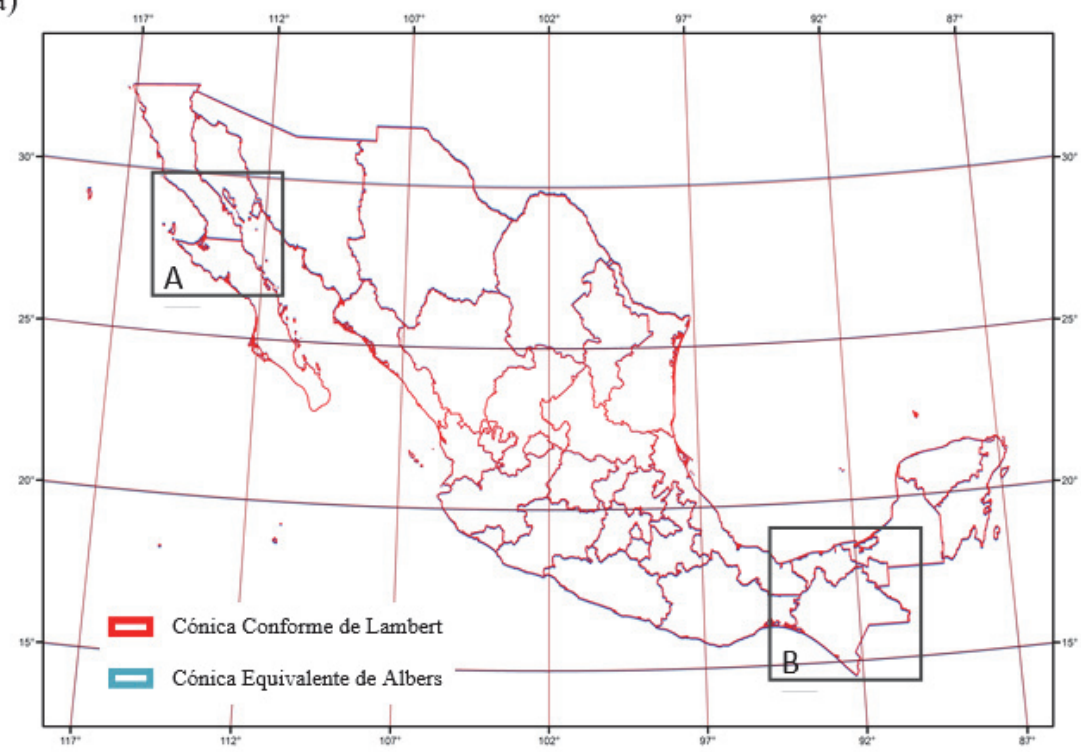

b)
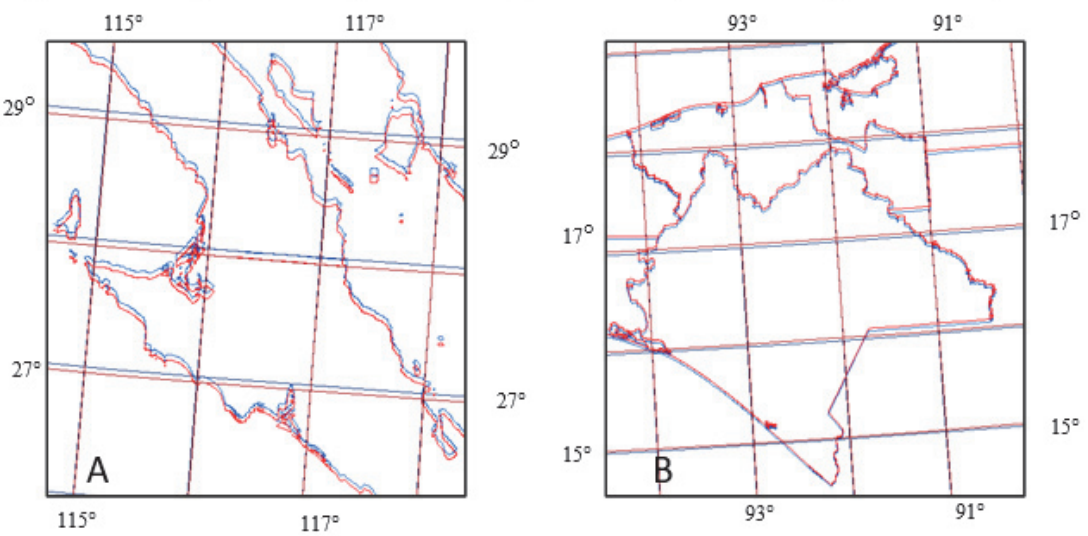

Figura 2. a) Comparación de la proyección de la República Mexicana en CCL y CEA. b) Comparación de estados del Norte y Sur de México.

Fuente: elaborado con base en procesamiento de archivos vectoriales en SIG.

A través de la superposición de la República Mexicana en las proyecciones CCL y CEA, tomando como referencia para la comparación la latitud y la longitud media del país, y considerando misma escala y coordenadas extremas, se observa que existe una diferencia mínima entre ambas proyecciones. La proyección CEA alarga ligeramente el territorio con dirección Norte-Sur, mientras que en el sentido Este- 
Oeste es poco visible la deferencia entre ambas. Al no haber una diferencia considerable, se puede afirmar que la CEA no distorsiona de manera relevante la forma del territorio.

Para representar a la República Mexicana en proyección CEA (Figura 3) se considera conveniente utilizar los parámetros que la Norma Técnica NTG-013-2006 de INEGI señala para la proyección CCL para México, ya que ambas son cónicas, secantes y normales:

- Paralelos Base: $29^{\circ} 30^{\prime} \mathrm{N}$ y $17^{\circ} 30^{\prime} \mathrm{N}$

- Meridiano central: $102^{\circ} \mathrm{W}$

- Latitud origen de la proyección: $12^{\circ} \mathrm{N}$

- Falso Norte: $0 \mathrm{~m}$

- Falso Este: 2,500,000 m

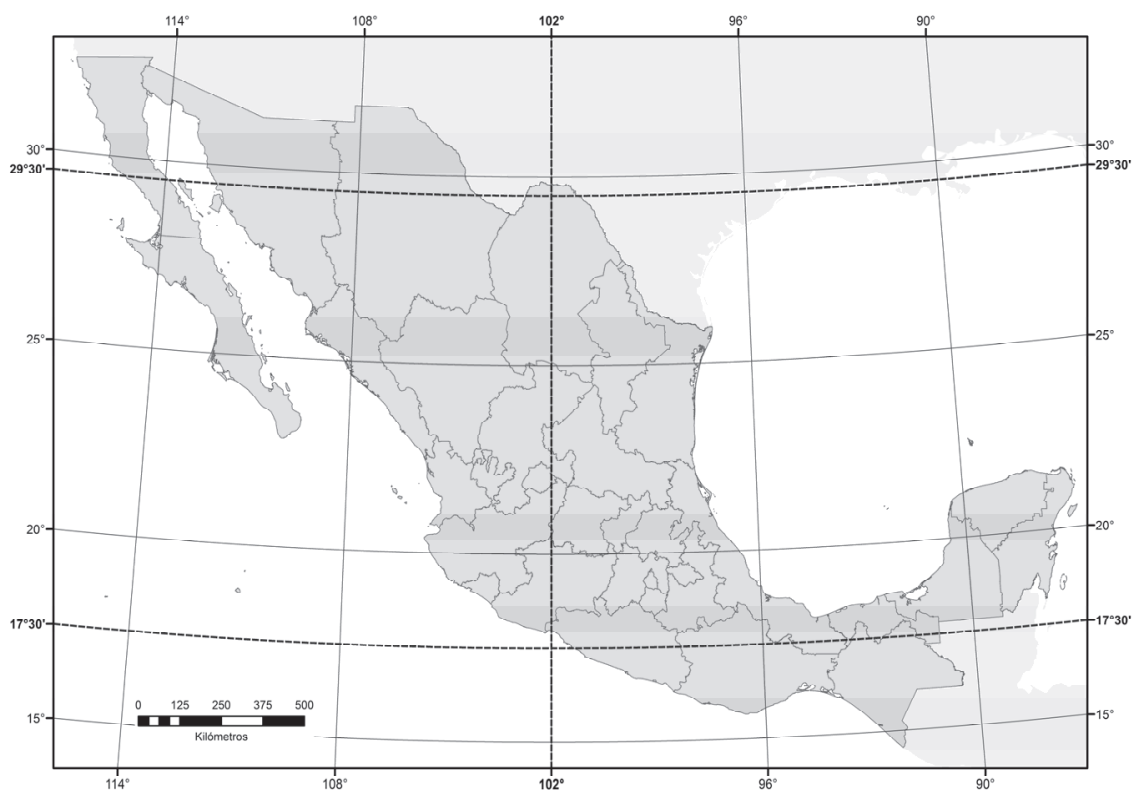

Figura 3. Parámetros para la representación de México en proyección CEA.

Fuente: elaborado con base en procesamiento de archivos vectoriales en SIG.

Debido a que los meridianos son perpendiculares a los paralelos, el factor de escala en un meridiano dado un punto es recíproco al factor de escala del paralelo; por lo que factor será mayor a la unidad sobre el meridiano y menor sobre el paralelo o viceversa (Adams, 1927). Esto se comprueba con los cálculos desarrollados para coordenadas de México proyectado en CEA (Tabla 2) (véase Figura 4). 
a)

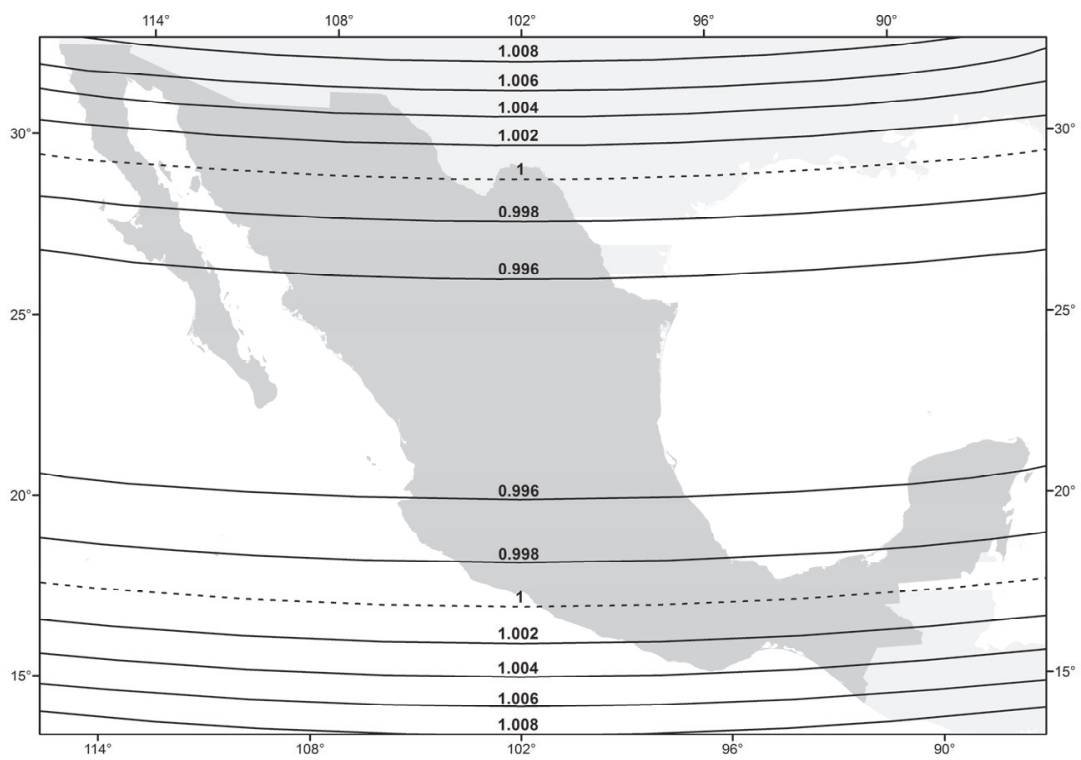

b)

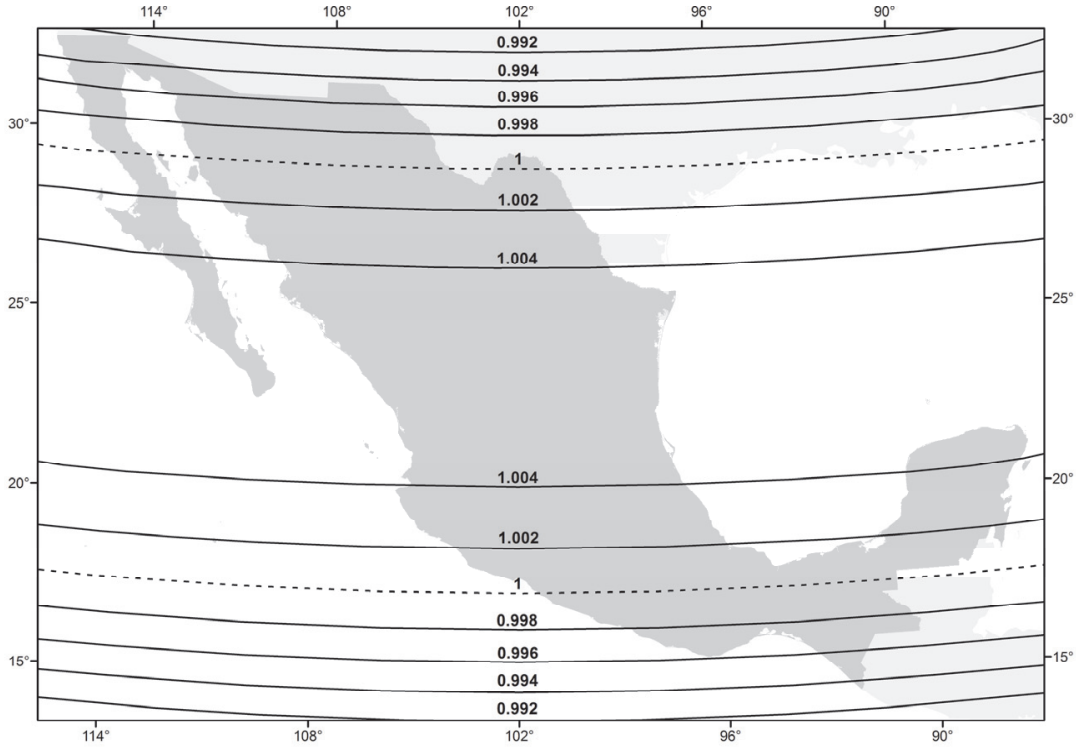

Figura 4. Factor de escala sobre proyección CEA para México. a) Factor de escala sobre paralelos, b) factor de escala sobre meridianos.

Fuente: elaborado sobre la base de INEGI, 2002; Córdoba, 1977 y procesamiento de archivos vectoriales en SIG. 
Tabla 2

Factor de escala sobre meridianos y paralelos en CEA Factor de escala sobre meridianos

\begin{tabular}{cccc}
\hline \multirow{2}{*}{ Latitud } & \multicolumn{2}{c}{ Arco de meridiano (1 ') } & \multirow{2}{*}{$\begin{array}{c}\text { Factor de } \\
\text { escala }\end{array}$} \\
\cline { 2 - 3 } & CEA & Elipsoide GRS 80 & 0.9927 \\
$32^{\circ} 30^{\prime}$ & 30.578161 & 30.804332 & 1.0000 \\
$29^{\circ} 30^{\prime}$ & 30.790009 & 30.790016 & 1.0055 \\
$23^{\circ} 30^{\prime}$ & 30.932599 & 30.764182 & 1.0000 \\
$17^{\circ} 30^{\prime}$ & 30.742995 & 30.742987 & 0.9936 \\
$1^{\circ} 30^{\prime}$ & 30.537795 & 30.734422 & \\
\hline
\end{tabular}

Factor de escala sobre paralelos

\begin{tabular}{cccc}
\hline \multirow{2}{*}{ Latitud } & \multicolumn{2}{c}{ Arco de paralelo (1') } & \multirow{2}{*}{$\begin{array}{c}\text { Factor de } \\
\text { escala }\end{array}$} \\
\cline { 2 - 3 } & CEA & Elipsoide GRS 80 & 1.0074 \\
\hline $32^{\circ} 30^{\prime}$ & 26.297728 & 26.104656 & 1.0000 \\
$29^{\circ} 30^{\prime}$ & 26.935079 & 26.935079 & 0.9946 \\
$23^{\circ} 30^{\prime}$ & 28.218033 & 28.372510 & 1.0000 \\
$1^{\circ} 30^{\prime}$ & 29.499842 & 29.499843 & 1.0064 \\
$1^{\circ} 30^{\prime}$ & 30.136234 & 29.943423 & \\
\hline
\end{tabular}

Nota: $\quad$ Medidas en metros.

Fuente: elaborado sobre la base de INEGI, 2002; Córdoba, 1977.

La relación inversa que existe entre el factor de escala entre meridianos y paralelos permite mantener la equivalencia de áreas a escala. En la Tabla 3 se muestra la comparación del cálculo de áreas en diferentes latitudes sobre la superficie proyectada en CEA y sobre el elipsoide.

Tabla 3

Comparación del cálculo de superficie sobre elipsoide y CEA

\begin{tabular}{ccc}
\hline & \multicolumn{2}{c}{ Superficie 1"latx 1"long } \\
\cline { 2 - 3 } Latitud & CEA & Elipsoide GRS80 \\
\hline $32^{\circ} 30^{\prime}$ & 2526.26929 & 2526.26828 \\
$29^{\circ} 30^{\prime}$ & 2605.42186 & 2605.42122 \\
$23^{\circ} 30^{\prime}$ & 2742.16132 & 2742.16138 \\
$17^{\circ} 30^{\prime}$ & 2849.15208 & 2849.15280 \\
$14^{\circ} 30$ & 2891.18829 & 2891.18933 \\
\hline
\end{tabular}

Nota: Medidas en metros cuadrados.

Fuente: elaborado sobre la base de INEGI, 2002; Córdoba, 1977. 
De manera gráfica, la Figura 5 muestra las indicatrices de Tissot en diversas latitudes; lo que se observa es que las variaciones de los semiejes de las elipses de deformación son mínimas.

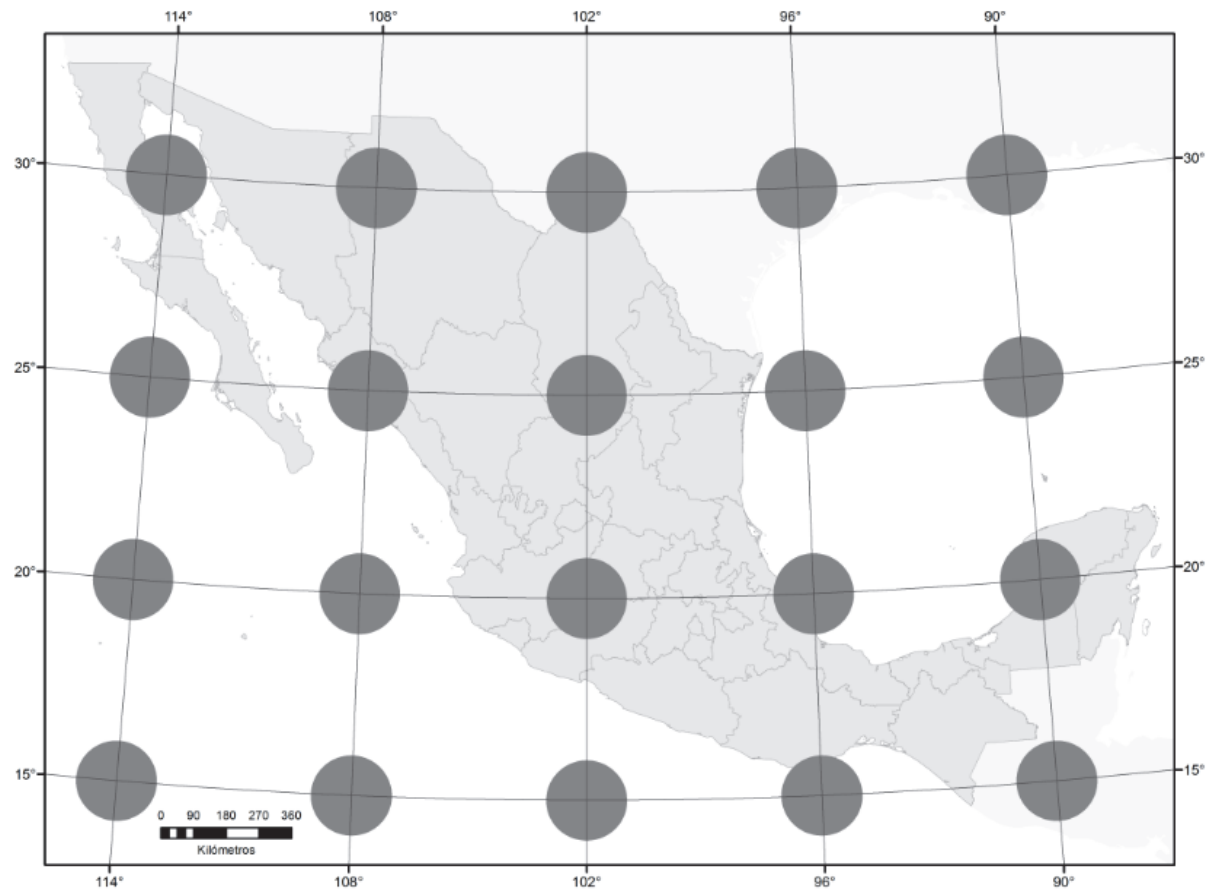

Figura 5. Indicatriz de Tissot sobre la proyección CEA adaptada para México.

Fuente: elaborado sobre la base de Córdoba, 1977 y procesamiento de archivos vectoriales en SIG.

La comparación de la variación del valor de superficie calculado en la CEA, con respecto a las diferentes proyecciones y el elipsoide que se emplean de manera oficial para la representación de México se muestran en la Tabla 4.

Las variaciones de las áreas que ocurren en cada proyección llevan a reflexionar que la realización de cálculos de superficies en proyecciones no equivalentes resultará en valores menos precisos e incluso desproporcionados con respecto a la realidad. De aquí la importancia de elegir una proyección adecuada para cada propósito. 
Tabla 4

Comparación de área de 1" de latitud por 1" de longitud en diferentes proyecciones y el elipsoide GRS 80

\begin{tabular}{|c|c|c|c|c|c|c|}
\hline & & $14^{\circ} 30^{\prime}$ & $17^{\circ} 30^{\prime}$ & $23^{\circ} 30^{\prime}$ & $29^{\circ} 30^{\prime}$ & $32^{\circ} 30^{\prime}$ \\
\hline$G R S$ & Área & $2,891.188$ & $2,849.152$ & $2,742.161$ & $2,605.422$ & $2,526.269$ \\
\hline \multicolumn{7}{|l|}{80} \\
\hline \multirow[t]{2}{*}{ UTM } & Área & $2,896.094$ & $2,853.318$ & $2,745.131$ & $2,607.042$ & $2,527.196$ \\
\hline & Diferencia & 4.905 & 4.166 & 2.970 & 1.620 & 0.927 \\
\hline \multirow[t]{2}{*}{$C C L$} & Area & $2,930.214$ & $2,849.151$ & $2,712.372$ & $2,605.423$ & $2,562.030$ \\
\hline & Diferencia & 39.025 & -0.001 & -29.789 & 0.001 & 35.761 \\
\hline \multirow[t]{2}{*}{$C E A$} & Área & $2,891.189$ & $2,849.153$ & $2,742.161$ & $2,605.421$ & $2,526.268$ \\
\hline & Diferencia & 0.001 & 0.001 & 0.000 & -0.001 & -0.001 \\
\hline
\end{tabular}

Notas:

1. Medidas en metros cuadrados.

2. Longitud de referencia: meridiano $102^{\circ} \mathrm{W}$.

3. La diferencia está calculada en relación a la superficie sobre el elipsoide.

Fuente: elaborado sobre la base de INEGI, 2002; Córdoba, 1977.

\section{Cartografía nacional equivalente en escala 1:500,000}

Con la finalidad de realizar una cartografía equivalente a escala nacional, se propone la separación del territorio en 45 cartas escala 1:500,000. Para esta cartografía, el territorio nacional puede dividirse en franjas de $6^{\circ}$ longitud, cada franja mantiene un meridiano central, de manera que se emplean seis meridianos centrales que coinciden con los utilizados en la proyección UTM para México $87^{\circ}, 93^{\circ}, 99^{\circ}, 105^{\circ}$, $111^{\circ}, 117^{\circ} \mathrm{W}$ (Figura 6).

El uso de franjas permite que la posición de las cartas permanezca muy cercana a la horizontal, de otra manera, en el caso de emplear un solo meridiano central, las cartas que se ubican en los extremos longitudinales del país presentarían una inclinación poco convencional. La discontinuidad del territorio segmentado en franjas para la obtención de las cartas no representa cambios en el factor de escala indicado en la Figura 4; de esta manera, dado que la escala varía sólo latitudinalmente, puede posibilitarse la continuidad por medio de cálculos matemáticos. Esta característica supone una ventaja con respecto a la cartografía UTM debido a que el factor de escala en dicha proyección aumenta tanto en sentido latitudinal como longitudinal y la unión de cartas es menos accesible. 


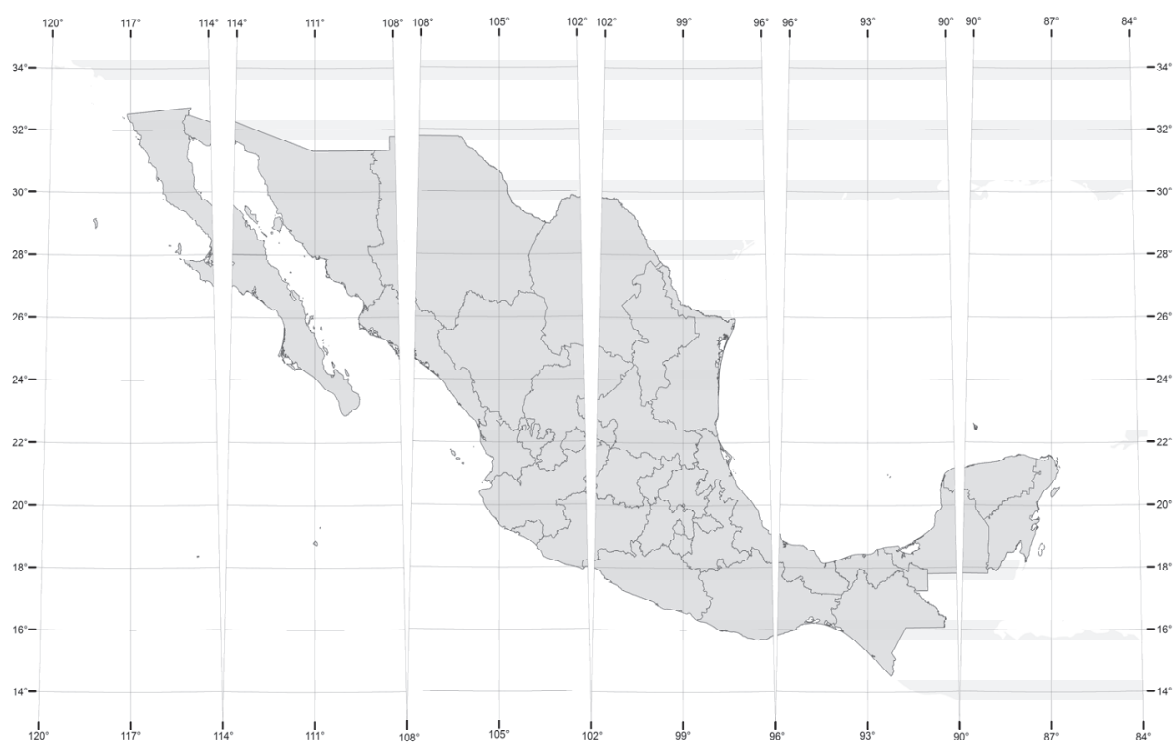

Figura 6. Representación de México en proyección CEA discontinua con seis meridianos centrales.

Fuente: elaborado con base en procesamiento de archivos vectoriales en SIG.

La Figura 7 muestra la división de las 45 cartas. El formato para su nomenclatura es derivado del que establece INEGI en la Norma Técnica NTG-013-2006, donde se señala que las dimensiones de una carta escala $1: 500,000$ son $2^{\circ}$ latitud por $3^{\circ}$ longitud, sin embargo, algunas cartas cubren hasta $2^{\circ} 30^{\prime}$ latitud y $3^{\circ} 30^{\prime}$ longitud debido a la configuración de la línea de costa del territorio. Las claves de las cartas se asignaron conforme a la Norma Técnica NTG-008-2005 División y Nomenclatura de los Conjuntos de Datos Espaciales por Escala emitida por el INEGI (2005), que indica que el nombre de las cartas se determina, por orden de prioridad, con base en la localidad con mayor número de habitantes, rasgos hidrológicos y rasgos orográficos; en este caso, por la amplia cobertura de las cartas, todas se nombraron de acuerdo a la localidad más poblada. Por otra parte, se les asignó una clave formada por un número romano, que numera el renglón en el que se ubica la carta, se comienza a enumerar desde los $34^{\circ} \mathrm{N}$ hasta $\operatorname{los} 14^{\circ} \mathrm{N}$; asimismo se asignó una letra que señala el número de columna desde la $\mathrm{A}$ en los $117^{\circ} \mathrm{W}$ hasta la $\mathrm{J}$ en los $87^{\circ} \mathrm{W}$. Con el fin de reducir el número de cartas y evitar representaciones con un cubrimiento continental mínimo, en las cartas III-A, VIII-E y VII-J se insertaron los territorios insulares Isla Guadalupe, Isla Clarión e Isla Socorro, y Arrecife Alacranes, respectivamente. 


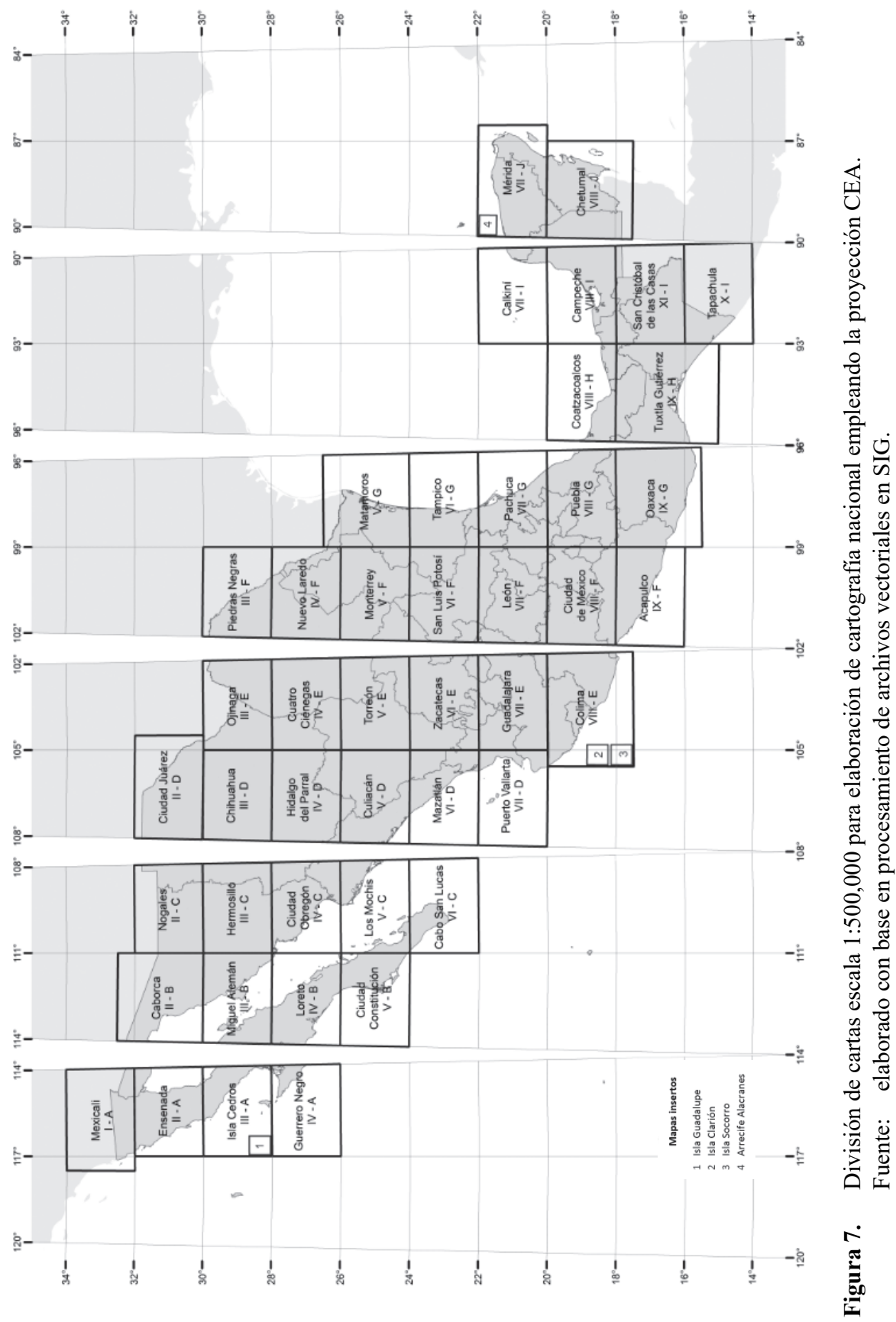




\section{Conclusiones}

En el presente trabajo se ha mostrado la posibilidad de representar el territorio mexicano de una manera satisfactoria con la proyección Cónica Equivalente de Albers (CEA); para ello, fue necesario tomar decisiones en cuanto a los parámetros matemáticos que deben aplicarse, dadas las condiciones de extensión latitudinal y longitudinal de México. Con esta optimización también se mantienen reducidas las deformaciones angulares.

La proyección CEA para México también mostró cualidades para su utilización en distintas escalas, lo que posibilita alcanzar una integración de la cartografía nacional. Con las proyecciones UTM y CCL, que tienen actualmente un uso regular en México, los estudios que requieran un análisis a diferentes escalas deben tomar decisión, ya sea por la preservación de una sola proyección y asumir el riesgo de usar inapropiadamente una de ellas, o bien, enfrentar el problema del cambio de sistema.

La proyección bajo estudio mostró una ventaja en el manejo del factor de escala en relación con la UTM. Es conocido que, en este último sistema, la escala varía tanto de forma latitudinal como longitudinal; los patrones de deformación de la CEA, en cambio, varían sólo en sentido latitudinal y será más preciso hacer mediciones de fenómenos que ocurren en diferentes longitudes, en particular si esas mediciones implican el cálculo de áreas. Asimismo, en los estudios de escala chica, mostró ventajas con respecto a la CCL, ya que, por sus propiedades, además de conservar la dimensión de las áreas, se observó que, al proyectar a México en la CEA bajo parámetros adecuados, esta no modifica sustancialmente la forma de los territorios.

Es así que una cartografía nacional equivalente para México permite a los usuarios dimensionar correctamente los fenómenos de interés; la escala 1:500,000, propuesta como primer acercamiento para la escisión de las cartas, posibilita la realización de cálculos analíticos y cartométricos sobre una base más precisa.

El presente trabajo no pretende descalificar la cartografía nacional que se realiza en México y que se ha practicado sobre bases fundamentadas; más bien, procura abonar en la discusión y ser de utilidad como producto de una investigación cartográfica que promueve el uso y entendimiento de las proyecciones cartográficas. La finalidad es contribuir con una alternativa, especialmente para los trabajos que no requieran emplear la cartografía oficial de México para mostrar sus resultados.

\section{Bibliografía}

Adams, O. (1927). Tables for Albers projection, US Government Printing Office, Estados Unidos. 
Alashaikh, A.; Bilani, H. y Alsalman, A. (2014), "Modified perspective cylindrical map projection”, Arabian Journal of Geosciences, Springer, 7(4):1559-1565.

Alonso, F. (1989). Apuntes de Cartografia, División de Ingeniería Civil, Topográfica y Geodésica, Universidad Nacional Autónoma de México, México.

Battersby, S. y Kessler, F. (2012). "Cues for Interpreting Distortion in Map Projections", Journal of Geography, 111(3):93-101.

Caire, J. (1974). "La proyección cartográfica para Petróleos Mexicanos", Boletín de la Asociación Mexicana de Geólogos Petroleros, 26(1-3), enero-marzo, México. (2002). Cartografía básica, Facultad de Filosofía y Letras, Universidad Nacional Autónoma de México, México.

Córdoba, G. (1977). "Control primario para la elaboración de cartas geográficas", tesis de licenciatura en Geografía, Facultad de Filosofía y Letras, Universidad Nacional Autónoma de México, México.

Dent, B. (1999). Cartography Thematic Map Design, Mc. Graw Hill, Estados Unidos.

Eckert, M. (1961). Cartografia, Unión Tipográfica Editorial Hispanoamericana, México.

Franco, S. y Valdez, M.A. (2003). Principios básicos de cartografía y cartografía automatizada, Universidad Autónoma del Estado de México, México.

Instituto Nacional de Estadística, Geografía e Informática (INEGI) (2002). Guía de proyecciones cartográficas, México.

- (2005), Norma Técnica NTG-008-2005 División y Nomenclatura de los Conjuntos de Datos Espaciales por escala, México.

(2006), Norma Técnica NTG-013-2006 Edición de Cartografía Topográfica, México.

Jenny, B. y Patterson, T. (2013). "Blending world map projections with Flex Projector", Cartography and Geographic Information Science, 40(4):289-296.

Jenny, B.; Patterson, T.; Petrovič, D. y Hurni, L. (2011). “A Polynomial Equation for the Natural Earth Projection", Cartography and Geographic Information Science, 38(4):363-372.

Kirtiloglu, O. (2010). "Distortion analysis of map projections", 10th International Multidisciplinary Scientific GeoConference SGEM2010, I:995-1002.

Oztug, I. (2015). "Quasi indicatrix approach for distortion visualization and analysis for map projections", International Journal of Geographical Information Science, 29(12):2295-2309.

Paquentín, E. (1956). "Proyección Cónica conforme de Lambert. Cálculo de la proyección respectiva de 5' en 5', Ingeniería Hidráulica de México, vol. X, julio-agosto-septiembre, pp. 57-66.

Patterson, T.; Šavrič, B. y Jenny, B. (2015). "Introducing the Patterson cylindrical projection", Cartographic Perspectives, 78:77-81. 
Raisz, E. (1985). Cartografía general, Omega, Barcelona.

Robinson, A.; Sale, R.; Morrison, J. y Muehrcke, P. (1987). Elementos de Cartografia, Omega, Barcelona.

Safari, A. y Ardalan, A. (2007). "New cylindrical equal area and conformal map projections of the reference ellipsoid for local applications", Survey Review, 39(304):132-144.

Salitchev, K. (1979). Cartografía, Editorial Pueblo y Educación, La Habana.

Streben, D. (2016). "An adaptable equal-area pseudoconic map projection", Cartography and Geographic Information Science, XVIII:338-345.

Šavrič, B. y Jenny, B. (2014). "A new pseudocylindrical equal-area projection for adaptive composite map projections", International Journal of Geographical Information Science, 28(12):2373-2389.

Šavrič, B.; Jenny, B.; Patterson, T.; Petrovič, D. y Hurni, L. (2011).” A polynomial equation for the Natural Earth projection", Cartography and Geographic Information Science, 38(4):363-372.

Snyder, J. (1987). Map projections, a working manual, U.S. Geological Survey Professional Paper 1395, Government Printing Office, Estados Unidos. (1997). Flattening the earth: two thousand years of map projections, University of Chicago Press, Estados Unidos.

Tyner, J. A. (2010). Principles of map design. Guilford Press, Estados Unidos. 